\title{
5-Demethylnobiletin is more effective than nobiletin in preventing AOM/DSS-induced colorectal carcinogenesis in ICR mice
}

\author{
Jia-Ching Wur,b,\#, Yen-Chen Tunga,\#, Yu-Nu Zheng ${ }^{\mathrm{c}}$, Mei-Ling Tsai ${ }^{\mathrm{c}}$, Ching-Shu Lai ${ }^{\mathrm{c}}$, \\ Chi-Tang $\mathrm{Ho}^{\mathrm{d}}$ and Min-Hsiung Pan ${ }^{\mathrm{a}, \mathrm{e}, \mathrm{f}^{*}}$
}

\begin{abstract}
anstitute of Food Science and Technology, National Taiwan University, Taipei 10617, Taiwan
bepartment of Environmental and Occupational Health, National Cheng Kung University Medical College, Tainan 704, Taiwan

${ }^{\mathrm{c}}$ Department of Seafood Science, National Kaohsiung Marine University, Kaohsiung 811, Taiwan

dDepartment of Food Science, Rutgers University, New Brunswick, NJ 08901, USA

eDepartment of Medical Research, China Medical University Hospital, China Medical University, Taichung 40402, Taiwan

fDepartment of Health and Nutrition Biotechnology, Asia University, Taichung, Taiwan

\#These authors contributed equally to this work

${ }^{*}$ Corresponding author: Min-Hsiung Pan, Institute of Food Science and Technology, National Taiwan University, No. 1, Section 4, Roosevelt Road, Taipei 10617, Taiwan. Tel: +886 2 33664133; Fax: +886-2-33661771; E-mail: mhpan@ntu.edu.tw

DOI: $10.31665 /$ JFB.2018.2144

Received: January 15, 2018; Revised received \& accepted: February 19, 2018

Citation: Wu, J.-C., Tung, Y.-C., Zheng, Y.-N., Tsai, M.-L., Lai, C.-S., Ho, C.-T., and Pan, M.-H. (2018). 5-Demethylnobiletin is more effective than nobiletin in preventing AOM/DSS-induced colorectal carcinogenesis in ICR mice. J. Food Bioact. 2: 98-103.
\end{abstract}

\begin{abstract}
Nobiletin and 5-demethylnobiletin as polymethoxyflavone and hydroxy polymethoxyflavone, especially, have been reported to exhibit various beneficial biological activities for human health. In this study, the effects of NOB and 5-demethylnobiletin (DMNB), on azoxymethane/dextran sodium sulfate (AOM/DSS)-induced colorectal carcinogenesis in Institute for Cancer Research (ICR) mice were compared. We found that NOB and DMNB significantly alleviated the weight loss, colon length and reduced colon tumor formation in AOM/DSS-induced colorectal carcinogenesis mice. At the molecular level, our results from western blot and polymerase chain reaction (PCR) analysis showed that $0.025 \%$ of NOB and DMNB presented an anti-inflammation property by reducing cyclooxygenase-2 (COX-2) and interleukin 6 (IL-6) expression. Taken together, these results demonstrate that DMNB had a better chemo-preventive efficacy than NOB in AOM/DSS-induced colorectal carcinogenesis in ICR mice model.
\end{abstract}

Keywords: Nobiletin; 5-Demethylnobiletin; Colorectal carcinogenesis; inflammation; Chemoprevention.

\section{Introduction}

Colorectal cancer (CRC) is one of the most common encountered cancers worldwide (Torre, Bray et al. 2015). Diet, lack of physical activity, family history of sporadic CRC and disease duration are risk factors of CRC development (Mandal 2017). Nowadays, chronic inflammation is recognized as a major risk factor for $\mathrm{CRC}$ development and progression and the progressive colorectal tumors are also associated with high levels of inflammation environment(Terzić, Grivennikov et al. 2010, Mandal 2017, Ray, Berggren et al. 2017). Moreover, chronic inflammation involves in several immune cell, cytokine, and proinflammatory mediators during the process of carcinogenesis (Mandal 2017). The proinflammatory cytokine interleukin 6 (IL-6) has been identified as a major regulator of inflammatory colon diseases and is correlated with CRC development, tumor metastasis, and reduced survival rate (Waldner, Foersch et al. 2012, Kakourou, Koutsioumpa et al. 2015, Wang, Wu et al. 2015). Moreover, inflammation-associated enzymes, such as cyclooxygenase-2 (COX-2), contribute to colitis-related colon tumorigenesis by production of prostaglandin E2 $\left(\mathrm{PGE}_{2}\right)$ and can be upregulated by azoxymethane/dextran sodium sulfate (AOM/DSS)-induced CRC in animal model that is a commonly used platform for colitis-related carcinogenesis intervention study (Snider, Bialkowska et al. 2016). Therefore, suppress severe 
(A)<smiles>COc1ccc(-c2cc(=O)c3c(OC)c(OC)c(OC)c(OC)c3o2)cc1OC</smiles>

(B)<smiles>COc1ccc(-c2cc(=O)c3c(O)c(OC)c(OC)c(OC)c3o2)cc1OC</smiles>

Figure 1. Structures of (A) nobiletin and (B) 5-demethylnobiletin.

inflammation responses in CRC development could be retarded the progressive of CRC.

Nobiletin $\left(5,6,7,8,3^{\prime}, 4^{\prime}\right.$-hexamethoxyflavone,NOB)(Figure 1A) is one of the major polymethoxyflavones in citrus peel and has gained increasing interest due to its beneficial properties including anti-inflammation, anti-cancer, and human health-promoting effects (Wu, Song et al. 2015, Li, Wang et al. 2017, Zheng, Bu et al. 2017). The compound 5-demethylnobiletin (5-hydroxy$6,7,8,3^{\prime}, 4^{\prime}$-pentamethoxyflavone, DMNB) (Figure 1B) is a hydroxy polymethoxyflavone, which is also found in citrus peel and/or in aged tangerein peels. Both NOB and DMNB have shown anti-cancer effects, and regulate lipid metabolism (Chen, Wang et al. 2015, Tung, Li et al. 2016). NOB has shown its effect on CRC, however, the role of DMNB on CRC has not yet been investigated ( $\mathrm{Wu}$, Song et al. 2015). In this study, for the first time, chemo-preventive effect of NOB and DMNB, in AOM/ DSS-induced colitis-associated carcinogenesis in ICR mice were investigated and offer possible molecular mechanism of their action suggested.

\section{Materials and methods}

\subsection{Reagents}

The NOB and DMNB were obtained from Florida Flavors Company (Lakeland, FL, USA). The purity of NOB and DMNB was determined to be higher than $95 \%$ by high-performance liquid chromatography (HPLC).

\subsection{Animals and experimental procedure}

Male Institute for Cancer Research (ICR) mice of 4-6 weeks of age were purchased from the BioLASCO experimental animal center (Taiwan Co., Taipei, Taiwan). All animals were housed under a controlled atmosphere $\left(25 \pm 1{ }^{\circ} \mathrm{C}, 50 \%\right.$ relative humidity, $12 \mathrm{~h}$ light/ $12 \mathrm{~h}$ dark cycle) and fed a standard AIN-76 diet and tap water ad libitum at all times. All experimental protocols used in these animal experiments were approved by the Institutional Animal Care and Use Committee (IACUC 0101-AAAP-09) of National Kaohsiung Marine University.

The animal experimental design in this study followed our previous study (Figure 2) (Lai, Yang et al., 2017). Briefly, all mice were randomly divided into six groups. Mice fed with normal diet and water as the control group. Mice in AOM/DSS treatment groups were administered AOM at a dose of $10 \mathrm{mg} / \mathrm{kg}$ via an intraperitoneal injection twice, followed by $2 \%$ DSS in drinking water as the AOM/DSS group. The NOB and DMNB treatment groups were fed diets containing 0.01 or $0.025 \%$ NOB and DMNB, respectively. At 15 weeks, all mice were euthanized by $\mathrm{CO}_{2}$ asphyxiation for further examination of molecular and histopathological changes in the colon tissues. The liver, spleen, and kidneys were

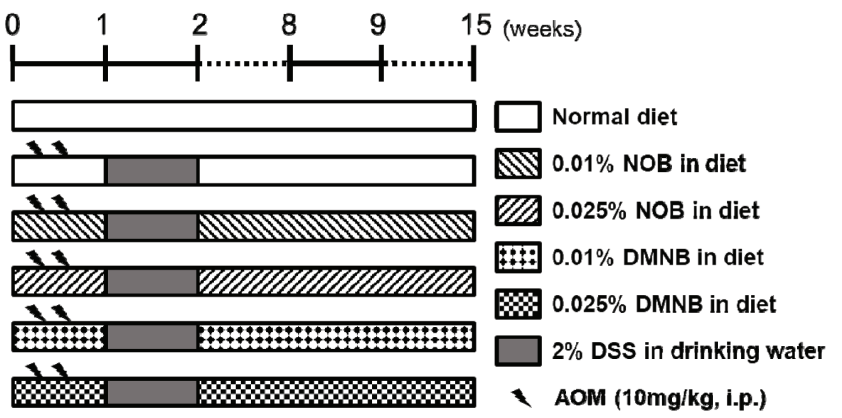

Figure 2. Experimental design of AOM/DSS-induced colorectal tumorigenesis in male ICR mice for 15 weeks. Effects of NOB or DMNB prevented AOM/DSS-induced colorectal tumorigenesis.

also immediately collected and weighed.

\subsection{Reverse transcription polymerase chain reaction}

Total RNA was extracted from the colonic mucosa using the TRIzol reagent (Invitrogen) according to the supplier's protocol. The complementary DNA (cDNA) was synthesized with $1 \mathrm{mg}$ total RNA, using SuperScript II RNA reverse transcriptase (RT) (Invitrogen). In the RT-PCR analysis, specific primers were designed to target IL- 6 and COX-2. The RT-PCR primers used in this experiment are as follows:

IL-6, 5'-TGGAGTACCATAGCTACCTGGAG-3', and 5'-TCCTTAGCCACTCCTTCTGTG-3'; COX-2, 5'-GGAGAGACTATCAAGATAGTGATC-3' and 5'-ATGGTCAGTAGACTTTTACAGCTC-3'; and GAPDH, 5'-TCAACGGCACAGTCAAGG-3' and 5'-ACTCCACGACATACTCAGC-3'. The PCR products were separated by electrophoresis on a $2 \%$ agarose gel and visualized using ethidium bromide (EtBr) nucleic acid staining (Yeastern Biotech, Taipei, Taiwan).

\subsection{Immunohistochemical analysis}

Colon tissues were fixed overnight in $10 \%$ buffered formalin and embedded in paraffin. Sections of 3- $\mu$ m thickness were incubated with $0.3 \%$ hydrogen peroxide to quench the endogenous peroxidase activity and were then heated in $10 \mathrm{mM}$ citrate buffer $(\mathrm{pH}$ 6.0 ) in a microwave oven for 10 min twice. Sections were then incubated with IL-6 primary antibody for $1 \mathrm{~h}$ at room temperature. Sections were incubated with a biotin-conjugated horseradish peroxidase secondary antibody, and 3,3'-diaminobenzidine tetrahydrochloride was used as the substrate.

\subsection{Western blot analysis}

For protein analyses, scraped colon mucosa tissue was homogenized with lysis buffer (50 mM Tris- $\mathrm{HCl}, 1 \mathrm{mM} \mathrm{NaF}, 150 \mathrm{mM}$ $\mathrm{NaCl}, 1 \mathrm{mM}$ ethylene glycol tetraacetic acid (EGTA), $1 \mathrm{mM}$ phenylmethanesulfonyl fluoride, $1 \% \mathrm{NP}-40$, and $10 \mu \mathrm{g} / \mathrm{mL}$ leupeptin) on ice for $30 \mathrm{~min}$, followed by centrifugation at $12,000 \times \mathrm{g}$ for $30 \mathrm{~min}$ at $4{ }^{\circ} \mathrm{C}$ to extract total protein. The $50-\mu \mathrm{g}$ protein samples were resolved by sodium dodecyl sulfate (SDS)-polyacrylamide gel electrophoresis, and the gel was electroblotted onto 0.45 $\mu \mathrm{m}$ polyvinylidene difluoride membrane. The membranes were blocked with $1 \%$ bovine serum albumin (BSA) solution and im- 
Table 1. Effects of NOB or DMNB on body weight and relative organ weight in AOM/DSS-induced CRC model

\begin{tabular}{lllll}
\hline \multirow{2}{*}{ Groups } & Body Weight $(\mathbf{g})$ & \multicolumn{2}{l}{ Relative organ weight (g) } \\
\cline { 3 - 5 } & & Liver & Kidney & Spleen \\
\hline Control & $50.7 \pm 3.3^{\mathrm{a}}$ & $2.27 \pm 0.16$ & $0.85 \pm 0.10$ & $0.12 \pm 0.04$ \\
AOM/DSS & $43.3 \pm 3.3^{\mathrm{b}}$ & $2.25 \pm 0.36$ & $0.84 \pm 0.09$ & $0.23 \pm 0.09 *$ \\
AOM/DSS + 0.01\% NOB & $46.8 \pm 4.8^{\mathrm{c}}$ & $2.12 \pm 0.31$ & $0.85 \pm 0.07$ & $0.13 \pm 0.03$ \\
AOM/DSS + 0.025\% NOB & $48.8 \pm 3.7^{\mathrm{ac}}$ & $2.16 \pm 0.22$ & $0.86 \pm 0.10$ & $0.15 \pm 0.08$ \\
AOM/DSS + 0.01\% DMNB & $47.6 \pm 4.6^{\mathrm{ac}}$ & $2.09 \pm 0.26$ & $0.85 \pm 0.13$ & $0.13 \pm 0.03$ \\
AOM/DSS + 0.025\% DMNB & $48.2 \pm 3.2^{\mathrm{ac}}$ & $2.10 \pm 0.22$ & $0.84 \pm 0.09$ & $0.13 \pm 0.05$ \\
\hline
\end{tabular}

The data of body weight are expressed as the mean \pm SD $(n=12)$ and were statistically analyzed using a one-way ANOVA followed by Duncan's multiple-range test. Different letters $(a-b)$ represent significant differences among groups, $p<0.05$. The data of relative organ weight performed by student's t-tests, ${ }^{*} p<0.05$, compared with control group.

munoblotted overnight at $4{ }^{\circ} \mathrm{C}$ with a primary antibody followed by a secondary antibody conjugated with horseradish peroxidase and visualized using enhanced chemiluminescence (ECL; Amersham Bioscience, Piscataway, NJ, USA). The densities of the bands were quantified using a densitometer (AlphaImagerTM 2200 System Alpha densitometer, Innotech Corporation, San Leandro, CA, USA).

\subsection{Statistical analysis}

All data were expressed as mean $\pm \mathrm{SD}$ for the indicated number of independently performed experiments. Statistical differences were determined using student's $t$-tests, one-way analysis of variance (ANOVA), and Duncan's multiple range tests using the SAS statistical software package (version 8.0). Differences of a $p$-value < 0.05 were considered statistically significant.

\section{Results and discussion}

\subsection{DMNB exhibited higher activity in preventing colonic tumor formation than NOB in an AOM/DSS-induced CRC model}

The AOM/DSS-induced colitis-associated colorectal tumorigenesis model is a recognized experimental model for investigating CRC development, and it has been considered an effective platform for evaluating the chemopreventive and chemotherapeutic activity of drugs and phytochemicals (Neufert, Becker et al. 2007, Lai, Yang et al. 2017). To evaluate and compare the effects of NOB and DMNB on AOM/DSS-inducedCRC model, we design the experimental model of AOM/DSS and NOB or DMNB treatment as shown in Figure 2. A previous study showed that $0.05 \%$ of NOB could alleviate the weight loss and decrease colon tumor incidence in vivo ( $\mathrm{Wu}$, Song et al. 2015). In this study, we found that the body weight decrease was accompanied with tumor formation in the AOM/DSS group $(43.3 \pm 3.3$ g) compared with the control group $(50.7 \pm 3.3 \mathrm{~g})$ Mice were fed with NOB or DMNB to retard body weight loss caused by AOM/ DSS. The relative organ weight in each group only the spleen weight of AOM/DSS group was heavier than other groups, due to the DSS cause inflammation in spleen (Perše and Cerar 2012) (Table 1)

Table 2 shows that AOM/DSS treatment significantly reduced the colon length, which was also observed in several other studies (Neufert, Becker et al. 2007, Chou, Suh et al. 2017, Zhang, $\mathrm{Su}$ et al. 2018). Dietary supplementation with NOB and DMNB restored the colon length $(\mathrm{mm})$ to $78.3 \pm 14.3$ and $90.6 \pm 14.4$, and $81.2 \pm 10.2$ and $101.3 \pm 11.4$, at 0.01 and $0.025 \%$ NOB or DMNB, respectively, compared to $67.4 \pm 6.0$ in the AOM/DSStreated group. We also observed that the AOM/DSS treatment group showed $100 \%$ tumor incidence; the total number of tumors in the AOM/DSS-treated group was $24.4 \pm 8.5$. The total number of colonic tumors was markedly and dose-dependently decreased by 0.01 and $0.025 \%$ NOB or DMNB consumption (10.2 $\pm 3.8,8.9 \pm 2.5$ and $8.0 \pm 3.6,7.6 \pm 3.1$, respectively) (Figure 3 $\&$ Table 2). These results revealed that both compounds could restore more colon length and decrease the number of tumor in AOM/DSS-induced colorectal tumorigenesis animal model.

Table 2. Effects of NOB or DMNB on the colon length and number of tumors in AOM/DSS-induced CRC model

\begin{tabular}{lllll}
\hline \multirow{2}{*}{ Groups } & Colon length $(\mathrm{mm})$ & \multicolumn{3}{c}{ NO. of Tumor/colon } \\
\cline { 3 - 5 } & & Small & Larger & Total \\
\hline Control & $87.1 \pm 5.7^{\mathrm{a}}$ & $1.1 \pm 1.1^{\mathrm{a}}$ & $0.0 \pm 0.0^{\mathrm{a}}$ & $1.1 \pm 1.1^{\mathrm{a}}$ \\
AOM/DSS & $67.4 \pm 6.0^{\mathrm{b}}$ & $10.4 \pm 6.2^{\mathrm{b}}$ & $14.0 \pm 10.4^{\mathrm{b}}$ & $24.4 \pm 8.5^{\mathrm{b}}$ \\
AOM/DSS + 0.01\% NOB & $78.3 \pm 14.3^{\mathrm{bc}}$ & $7.8 \pm 1.8^{\mathrm{ab}}$ & $2.4 \pm 2.8^{\mathrm{a}}$ & $10.2 \pm 3.8^{\mathrm{c}}$ \\
AOM/DSS + 0.025\% NOB & $90.6 \pm 14.4^{\mathrm{ac}}$ & $7.4 \pm 2.1^{\mathrm{c}}$ & $1.4 \pm 2.0^{\mathrm{a}}$ & $8.9 \pm 2.5^{\mathrm{c}}$ \\
AOM/DSS + 0.01\% DMNB & $81.2 \pm 10.2^{\mathrm{ac}}$ & $6.9 \pm 2.8^{\mathrm{c}}$ & $1.1 \pm 1.4^{\mathrm{a}}$ & $8.0 \pm 3.6^{\mathrm{c}}$ \\
AOM/DSS + 0.025\% DMNB & $101.3 \pm 11.4^{\mathrm{c}}$ & $6.6 \pm 1.9^{\mathrm{c}}$ & $1.0 \pm 2.0^{\mathrm{a}}$ & $7.6^{\mathrm{c}} \pm 3.1^{\mathrm{c}}$ \\
\hline
\end{tabular}

The data of body weight are expressed as the mean $\pm S D(n=12)$ and were statistically analyzed using a one-way ANOVA followed by Duncan's multiple-range test. Different letters (a-b) represent significant differences among groups, $p<0.05$. 


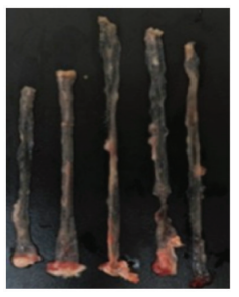

Control

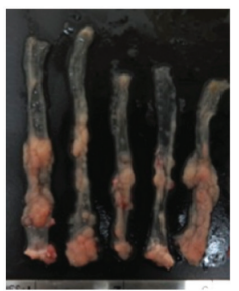

Positive

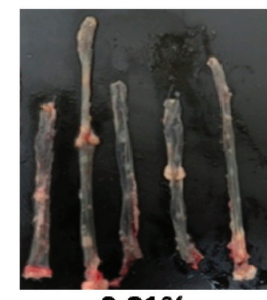
$0.01 \%$

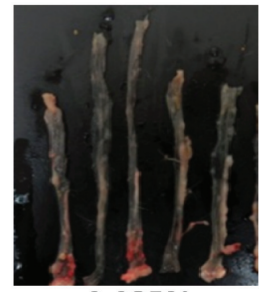

NOB

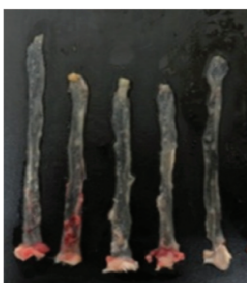

$0.01 \%$

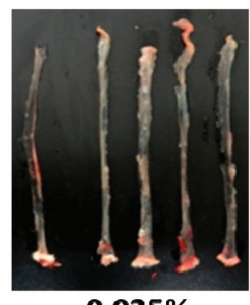

$0.025 \%$
DMNB

Figure 3. Morphology of colonic mucosal surface of AOM/DSS-treated mice. (A) control group (B) AOM/DSS group (C) NOB and (D) DMNB group. Positive: AOM/DSS.

\subsection{Orally administrated DMNB significantly prevented AOM/ DSS-induced inflammation through decreased IL-6 and COX-2 ex- pression levels in colonic mucosal tissue}

Growing evidence indicates that chronic inflammation is a major and determining factor in initiation and promotion of CRC (Do, Hwang et al. 2016). For example, IL-6 is an important cytokine in the development of colitis and CRC by acting as a tumor-promoting factor and increasing the expression level of IL-6, which is correlated in AOM/DSS-treated rodents with colitis and patients with inflammatory bowel disease (Li, de Haar et al. 2010, Wang and Sun 2014, Chen, Ma et al. 2015). In this study, the results showed that NOB and DMNB significantly suppressed IL-6 expression in the colon tissue by immunohistochemical analysis. We also found that NOB and DMNB suppressed IL-6 protein and mRNA expression compared with AOM/DSS group (Figure 4). (Figures 5A \& 5C). Furthermore, COX-2, which is an inflammation biomarker that contributes to colon tumorigenesis by the production of prostaglandin E2 and our previous study showed that AOM/DSS treated mice had higher protein level of COX-2 (Figure 5B \& 5C)(Takahashi and Wakabayashi 2004, Lai, Yang et al. 2017). Wu et al. (2017) showed that a nobiletin mixture containing three metabolites (3'-demethylnobiletin, 4'-demethylnobiletin and $3^{\prime}, 4^{\prime}$ didemethylnobiletin) suppressed colitisassociated colon carcinogenesis by down-regulating iNOS in AOM/DSS induced CRC model (Wu, Song et al. 2017). Another study showed that hydroxylated PMFs decreased the number of aberrant crypt foci in colonic tissues by significantly decreasing the levels of inducible nitric oxide synthase, COX-2 in AOMinduced colonic tumorigenesis model (Lai, Tsai et al. 2011). In this study, AOM/DSS significantly induced COX-2 expression, and it was suppressed by mice fed with NOB or DMNB. Interestingly, DMNB group reduced more COX-2 expression than NOB group. Consequently, our results demonstrated that the chemopreventive effect of DMNB was more potent than that of NOB in this chemopreventive effect and was associated with a decreased inflammation and tumor formation in the AOM/DSS-induced
(A)

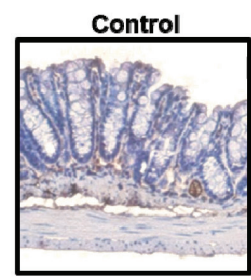

Positive

(AOM/DSS)
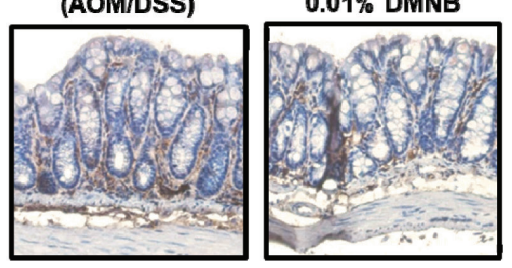

$0.025 \%$ DMNB

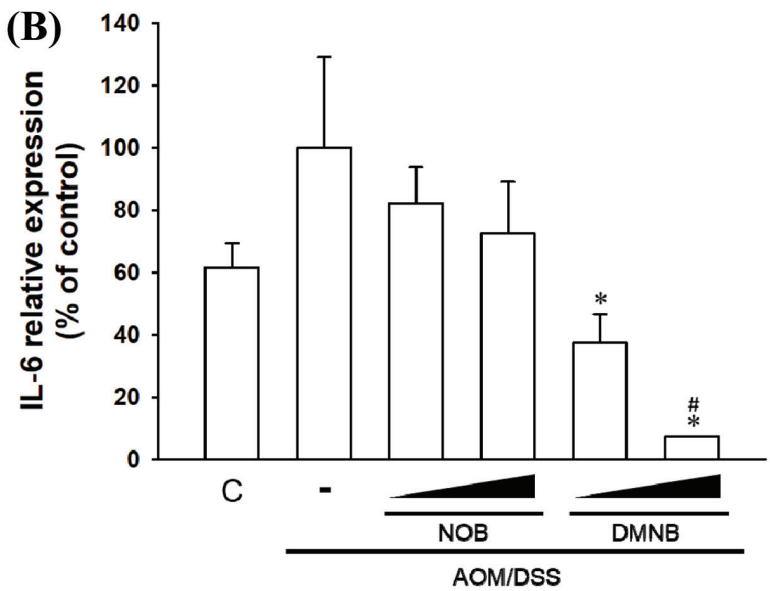

Figure 4. Inhibitoy effect of NOB and DMNB on AOM/DSS-induced IL-6 expression and distribution in colorectal tissue. (A) Colonic tissues were embedded in paraffin and visualized by immunohistochemical (IHC) analysis using IL-6 antibody demonstrated as dark stain. (B) Quantification of IL-6-labeling index (\%) was determined by ImageJ (National Institute of Health). ${ }^{*} p<0.05$, compared with control group. \#p<0.05, compared with AOM/DSS group.

CRC model.

\section{Conclusion}

The dietary administration of NOB and DMNB suppressed AOM/ DSS-induced CRC by improving colon shortening, tumor formation, and inflammatory responses. More importantly, DMNB interfered with IL-6, and COX-2 expression and this could be a possible mechanism for better prevention against the CRC effect than NOB. These results show that DMNB has great potential as a novel chemopreventive agent that can be used in the prevention of colitis-associated CRC.

\section{Acknowledgments}

This study was supported by the Ministry of Science and Technol- 
(A)

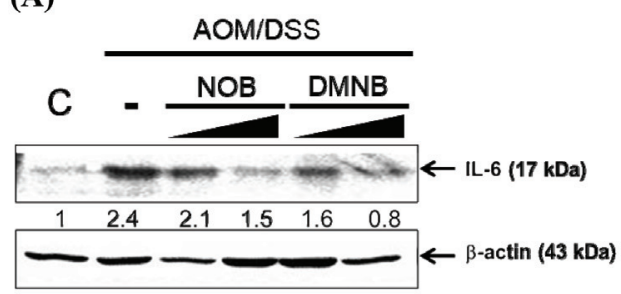

(B)

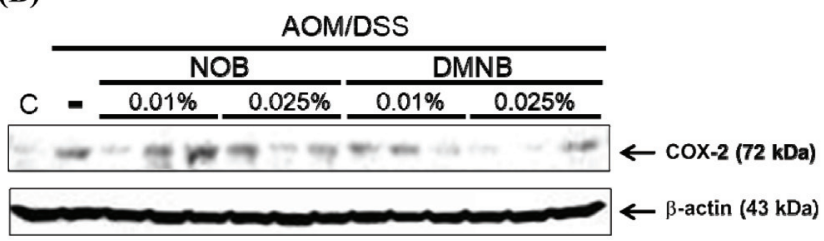

(C)

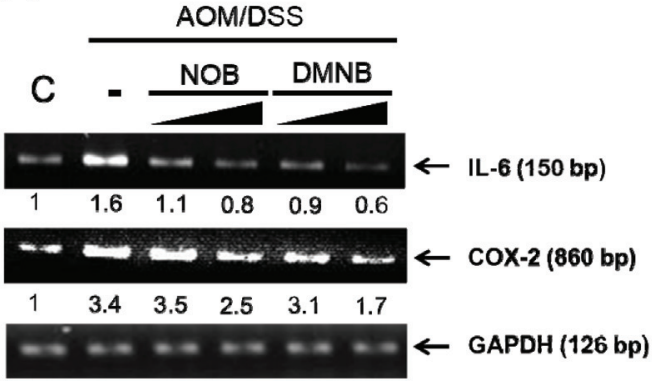

Figure 5. The NOB and DMNB significantly prevented AOM/DSS-induced inflammatory responses in colonic mucosa tissue. Total protein lysates from colonic mucosa detected the expression level of IL-6 (A) and COX-2 (B) by western blot. (C) Reverse transcriptional PCR analyzed colonic mucosa mRNA expression level of IL-6 and COX-2.

ogy [105-2320-B-002-031-MY3, 105-2628-B-002-003-MY3].

\section{Conflict of interest}

The authors declare no conflict of interest.

\section{References}

Chen, Y.K., Wang, H.C., Ho, C.T., Chen, H.Y., Li, S., Chan, H.L., Chung, T.W., Tan, K.T., Li, Y.R., and Lin, C.C. (2015). 5-Demethylnobiletin promotes the formation of polymerized tubulin, leads to G2/M phase arrest and induces autophagy via JNK activation in human lung cancer cells. J Nutr Biochem. 26(5): 484-504.

Chen, Y.Y., Ma, Z.B., Xu, H.Y., Shi, L.J., Li, D.Y., Sun, L.Y., Yin, X.H., Sang, G.Y., Xu, D., Tang, Y.H., Wang, X., Li, P., Wu, F., and Zhou, J. (2015). IL-6/ STAT3/SOCS3 signaling pathway playing a regulatory role in ulcerative colitis carcinogenesis. Int J Clin Exp Med. 8(8): 12009.

Chou, Y.C., Suh, J.H., Wang, Y., Pahwa, M., Badmaev, V., Ho, C.T., and Pan, M.H. (2017). Boswellia serrata resin extract alleviates azoxymethane $(\mathrm{AOM}) /$ dextran sodium sulfate (DSS)-induced colon tumorigenesis. Mol. Nutr. Food Res. 61(9): 1600984

Do, E.J., Hwang, S.W., Kim, S.Y., Ryu, Y.M., Cho, E.A., Chung, E.J., Park, S., Lee, H.J., Byeon, J.S., Ye, B.D., Yang, D.H., Park, S.H., Yang, S.K., Kim, J.H., and Myung, S.J. (2016). Suppression of colitis-associated carcinogenesis through modulation of IL-6/STAT3 pathway by balsalazide and VSL\# 3. J Gastroenterol Hepatol. 31(8): 1453-1461.
Kakourou, A., Koutsioumpa, C., Lopez, D.S., Hoffman-Bolton, J., Bradwin, G., Rifai, N., Helzlsouer, K.J., Platz, E.A., and Tsilidis, K.K. (2015). Interleukin- 6 and risk of colorectal cancer: results from the CLUE II cohort and a meta-analysis of prospective studies. Cancer Causes Control. 26(10): 1449-1460.

Lai, C.S., Yang, G., Li, S., Lee, P.S., Wang, B.N., Chung, M.C., Nagabhushanam, K., Ho, C.T., and Pan, M.H. (2017). 3'-Hydroxypterostilbene Suppresses Colitis-Associated Tumorigenesis by Inhibition of IL-6/STAT3 Signaling in Mice. J Agric Food Chem. 65(44): 9655-9664.

Lai, C.S., Tsai, M.L., Cheng, A.C., Li, S., Lo, C.Y., Wang, Y., Xiao, H., Ho, C.T., Wang, Y.J., and Pan, M.H. (2011). Chemoprevention of colonic tumorigenesis by dietary hydroxylated polymethoxyflavones in azoxymethane-treated mice. Mol Nutr Food Res. 55(2): 278-290.

Li, W., Wang, X., Zhi, W., Zhang, H., He, Z., Wang, Y., Liu, F., Niu, X., and Zhang, X. (2017). The gastroprotective effect of nobiletin against ethanol-induced acute gastric lesions in mice: impact on oxidative stress and inflammation. Immunopharmacol Immunotoxicol. 39(6): 354-363.

Li, Y., de Haar, C., Chen, M., Deuring, J., Gerrits, M.M., Smits, R., Xia, B., Kuipers, E.J., and van der Woude, C.J. (2010). Disease-related expression of the IL6/STAT3/SOCS3 signalling pathway in ulcerative colitis and ulcerative colitis-related carcinogenesis. Gut. 59(2): 227-235.

Mandal, P. (2018). Molecular signature of nitric oxide on major cancer hallmarks of colorectal carcinoma. Inflammopharmacology 26(2): 331-336.

Neufert, C., Becker, C., and Neurath, M.F. (2007). An inducible mouse model of colon carcinogenesis for the analysis of sporadic and inflammation-driven tumor progression. Nat Protoc. 2(8): 1998-2004.

Perše, M., and Cerar, A. (2012). Dextran sodium sulphate colitis mouse model: traps and tricks. J Biomed Biotechnol. 2012: 718617.

Ray, A.L., Berggren, K.L., Restrepo Cruz, S., Gan, G.N., and Beswick, E.J. (2018). Inhibition of MK2 suppresses IL-1 $\beta$, IL-6, and TNF- $\alpha$ dependent colorectal cancer growth. Int J Cancer 142 (8): 1702-1711.

Snider A.J., Bialkowska A.B., Ghaleb A.M., Yang V.W., Obeid L.M., Hannun Y.A. (2016) Murine Model for Colitis-Associated Cancer of the Colon. In: Proetzel G., Wiles M. (eds) Mouse Models for Drug Discovery. Methods in Molecular Biology, vol 1438. Humana Press, New York, NY, pp. 245-254.

Takahashi, M., and Wakabayashi, K. (2004). Gene mutations and altered gene expression in azoxymethane-induced colon carcinogenesis in rodents. Cancer Sci. 95(6): 475-480.

Terzić, J., Grivennikov, S., Karin, E., and Karin, M. (2010). Inflammation and colon cancer. Gastroenterology 138(6): 2101-2114, e2105.

Torre, L.A., Bray, F., Siegel, R.L., Ferlay, J., Lortet-Tieulent, J., and Jemal, A. (2015). Global cancer statistics, 2012. CA Cancer J Clin. 65(2): 87-108.

Tung, Y.C., Li, S., Huang, Q., Hung, W.L., Ho, C.T., Wei, G.J., and Pan, M.H. (2016). 5-Demethylnobiletin and 5-Acetoxy-6,7,8,3', $4^{\prime}$ pentamethoxyflavone Suppress Lipid Accumulation by Activating the LKB1-AMPK Pathway in 3T3-L1 Preadipocytes and High Fat Diet-Fed C57BL/6 Mice. J Agric Food Chem. 64(16): 3196-3205.

Waldner, M.J., Foersch, S., and Neurath, M.F. (2012). Interleukin-6-a key regulator of colorectal cancer development. Int J Biol Sci. 8(9): 1248.

Wang, S.W., and Sun, Y.M. (2014). The IL-6/JAK/STAT3 pathway: potential therapeutic strategies in treating colorectal cancer. Int J Oncol. 44(4): 1032-1040.

Wang, Z., Wu, P., Wu, D., Zhang, Z., Hu, G., Zhao, S., Lai, Y., and Huang, J. (2015). Prognostic and clinicopathological significance of serum interleukin-6 expression in colorectal cancer: a systematic review and meta-analysis. Onco Targets Ther. 8: 3793.

Wu, X., Song, M., Gao, Z., Sun, Y., Wang, M., Li, F., Zheng, J., and Xiao, H. (2017). Nobiletin and its colonic metabolites suppress colitis-associated colon carcinogenesis by down-regulating iNOS, inducing antioxidative enzymes and arresting cell cycle progression. J Nutr Biochem. 42: $17-25$.

Wu, X., Song, M., Wan, g.M., Zheng, J., Gao, Z., Xu, F., Zhang, G., and Xiao, H. (2015). Chemopreventive effects of nobiletin and its colonic metabolites on colon carcinogenesis. Mol Nutr Food Res. 59(12): 23832394.

Zhang, M.J., Su, H., Yan, J.Y., Li, N., Song, Z.Y., Wang, H.J., Huo, L.G., Wang, F., Ji, W.S., Qu, X.J., and Qu, M.H. (2018). Chemopreventive effect of Myricetin, a natural occurring compound, on colonic chronic in- 
flammation and inflammation-driven tumorigenesis in mice. Biomed Pharmacother. 97: 1131-1137.

Zheng, Y., Bu, J., Yu, L., Chen, J., and Liu, H. (2017). Nobiletin improves propofol-induced neuroprotection via regulating Akt/mTOR and TLR $4 / \mathrm{NF}-\mathrm{kB}$ signaling in ischemic brain injury in rats. Biomed Pharmacother. 91: 494-503. 